\title{
Effects of microgravity crystallization on a ligand-induced RNA crystal phase transition
}

\author{
Jason R. Stagno ${ }^{1}$, Ping $\mathrm{Yu}^{1}$, April Spinale ${ }^{2}$, Paul W. Todd ${ }^{3}$, Marc Giulianotti ${ }^{2}$, Yun-Xing Wang ${ }^{1}$ \\ ${ }^{1}$ Structural Biophysics Laboratory, Center for Cancer Research, National Cancer Institute, Frederick, MD, \\ 21702; ${ }^{2}$ Center for the Advancement of Science in Space, Melbourne, FL 32940; ${ }^{3}$ Techshot, Inc., \\ Greenville, IN 47124
}

Email Contact: jason.stagno@nih.gov

Virtually all biological processes are driven by the complex interactions among biomolecules. In order to understand the structural bases and time-courses for those interactions, one needs to determine their three-dimensional structures, as well as the changes of those structures over time. The frontier of structural biology is shifting toward the recording of time-resolved (TR) biological events - "molecular movies" that consist of many snapshots of entire molecular processes. Paramount to such four-dimensional crystallography is the use of X-ray free electron lasers (XFELs), whose ultra-brilliance and femtosecond pulse-rates enable room-temperature crystallographic studies on timescales much faster than biological reactions. In 2016, we reported the first diffusive mixing TR XFEL experiments on the adenine riboswitch RNA, demonstrating large conformational changes in crystallo upon ligand binding, accompanied by crystal phase transition. We found that the majority of crystals do not survive the transition, and for those that do, the diffraction data are very difficult to interpret. We posit that the "survival" of crystals that undergo phase transition is determined by the cooperativity - spatially and temporally - of the molecular changes throughout the lattice, which depends on 1) crystal plasticity (achieved in part using microcrystals), 2) crystal homogeneity (minimal variance in size and shape), and 3) crystal uniformity/quality (minimal variance throughout the lattice). To test this hypothesis, we will grow the crystals in a microgravity environment aboard the International Space Station (ISS) in order to minimize the imperfections and heterogeneity of the starting crystalline material, as many of these defects could be a direct result of forces imposed by terrestrial gravitation. We will perform largescale (multiple $10 \mathrm{~mL}$ ) batch crystallization experiments using a simple syringe-based mixing apparatus. In addition, we will conduct small-scale $(1 \mathrm{~mL})$ batch crystallization under varying conditions using the BISEP crystallization cassette and Advanced Space Experiment Processer 2 (ADSEP2) (Techshot, Inc). Identical experiments will be performed on the ground as controls. Upon return of the payload to Earth, crystals generated from both experiments will be used for time-resolved mixing experiments at an XFEL. 